\title{
The treatment of myocardial infarction through bone marrow mesenchymal stem cells transplantation combined with neonatal rat's cardiomyocytes
}

Xinqun Xu ${ }^{1}$, Yang Wang ${ }^{2}$, Yunfeng Wei ${ }^{1}$, Menghong Wang ${ }^{1}$, Zhiliang Zhang ${ }^{1}$, Jun Huang ${ }^{1}$, Junyi Zeng ${ }^{1}$, Guoqiu Ying $^{1}$

${ }^{I}$ Department of Cardiology, ${ }^{2}$ Institute of Urinary Surgery, the First Affiliated Hospital of NanChang University,Nanchang 330006, China

To observe the therapeutic effect of the transplatation of bone marrow mesenchymal stem cells (BMSCs) combined with neonatal rat's cardiomyocytes (NRCMs) on the myocardial infartion rats. Methods 1 . The BMSCs cells were collected from bone marrow of SD rat's femord bones,cultured.The fourth generation BMSCs were labelled with CM-Dil. 2. The NRCMs were collected from the heart of neonatal rats. 3.Experimental animals and groups:The rats were randomly divided into 4 groups after producting myocardial infarction. (1) Control group: The rats were received the PBS. (2) BMSCs group:The rats were received the BMSCs. (3) NRCMs group:The rats were received the NRCMs. (4) BMSCs+ NRCMs group:The rats were received the BMSCs+ NRCMs. 4. 4 weeks after operation, the heart function was assessed by echocardiography and the hemodymamic left ventricular measurements. The hearts were removed, to investigate the BMSCs survival, distribution, transfer, differentiation and the density capillary in infarction area by fluorescence microscope. Results 1.The heart function of rats assessed with echocardiography: Ejection fraction(\%)(BMSCs+ NRCMs group 47.81 \pm 3.61 vs BMSCs group $44.41 \pm 2.45$ vs $C$ group $40.52 \pm 2.61, P<0.05$, NRCMs group $43.64 \pm 3.81$ vs the others, $P>0.05$.). 2. The hemodymatic left ventricular measurements: Maximum left ventricular pressure rising rate $+\mathrm{dp} / \mathrm{dt}(\mathrm{mmHg} / \mathrm{s})$ BMSCs + NRCMs group $3444.23 \pm 327.45$ vs BMSCs group 3167.26 \pm 266.98 vs Control group 2879.78 $\pm 239.00, P<0.05, N R C M s$ group 2976.85 \pm 108.85 vs the others, $P>0.05$ ). Dropping rising -dp/dt ( $\mathrm{mmHg} / \mathrm{s})(B M S C s+$ NRCMs group 3156.60 \pm 297.73 vs BMSCs group 2892.38 \pm 226.18 vs Control group 2060. $60 \pm 281.40, P<0.05$, NRCMs group $2770.35 \pm 110.23$ vs the others, $P>0.05)$. 3 . The density of capillary in infarction area(n/HPF): (BMSCs+ NRCMs group 5.73 \pm 1.47 vs BMSCs group 3.93 \pm 0.91 vs Control group 2.04 $\pm 0.87, P<0.05$, NRCMs group $3.10 \pm 1.11$ vs the others, $P>0.05$ ). 4 . The positive CM-Dil cells were observed at infracted area in BMSCs group and BMSCs+ NRCMs group. The cells positive for both CM-Dil and cTnT were also observed in BMSCs group and BMSCs+ NRCMs group, it indicated that the transplanted BMSCs were able to survive and differentiate into cardiac-like cells. Conclusions BMSCs cotransplantation with NRCMs can obviously improve heart function, which may result from a better mircoenviroment and a stronger angiogenesis.

Keywords: bone marrow mesenchymal stem cells, neonatal rat's cardiomyocytes, myocardial infarction, cotransplantation Cell Research (2008) 18:s68. doi: 10.1038/cr.2008.158; published online 4 August 2008

Correspondence: Yunfeng Wei

E-mail: XXq9504@sohu.com 